\title{
Human Apolipoprotein A-IV
}

\author{
INTESTINAL ORIGIN AND DISTRIBUTION IN PLASMA
}

\author{
Peter H. R. Green, Robert M. Glickman, John W. Riley, and \\ ElaINe QUINET, Gastrointestinal Unit, Columbia University College of \\ Physicians i Surgeons, New York 10032
}

\begin{abstract}
A B S TRACT The role of the human intestine has been explored as a site of synthesis of apoA-IV, a major apoprotein of human intestinal triglyceride-rich lipoproteins. Intestinal biopsies were performed on normal volunteers while fasting and after lipid ingestion. Indirect immunofluorescence demonstrated a marked increase in immunofluorescence for apoA-IV during lipid absorption consistent with an increased intracellular content. ApoA-IV comprised $10-13 \%$ of chylomicron apoprotein and $24-30 \%$ of intestinal very low density lipoprotein (VLDL) as assessed by densitometry of sodium dodecyl sulfate gels of lipoproteins from chylous urine (mesenteric lymphatic-urinary fistula) and thoracic duct lymph (postoperative fistula). After one subject with chyluria ingested $40 \mathrm{~g}$ of corn oil, triglyceride excretion in urine was accompanied by an increased excretion of apoA-IV. $11.5 \mathrm{~g}$ of triglyceride and $81 \mathrm{mg}$ of apoA-IV were recovered in the urine. In chylous urine $56 \%$ of apoA-IV was in the triglyceriderich lipoproteins (chylomicrons and intestinal VLDL) and $44 \%$ in the $d>1.006-\mathrm{g} / \mathrm{ml}$ fraction.
\end{abstract}

Normal plasma apoA-IV was $15.7 \pm 0.9 \mathrm{mg} / \mathrm{dl}(n=14)$ whereas four subjects with abetalipoproteinemia had reduced levels $1.2,7.6,9.6$, and $8.3 \mathrm{mg} / \mathrm{dl}$, respectively. Lipid feeding in normal volunteers resulted in a rise in plasma apoA-IV $(16.1 \pm 0.7 \mathrm{mg} / \mathrm{dl}$ to $18.5 \pm 0.7 \mathrm{mg} / \mathrm{dl}$, $n=5, P<0.01$ ). In fasting plasma, $98 \%$ of apoA-IV was in the $d>1.21 \mathrm{gg} / \mathrm{ml}$ fraction. In lipemic plasma, $10 \%$ of apoA-IV was associated with triglyceride-rich lipoproteins and $90 \%$ with the $d>1.21-\mathrm{g} / \mathrm{ml}$ fraction. Agarose column chromatography of fasting plasma confirmed that the bulk of plasma apoA-IV is free, unassociated with lipoproteins.

These results demonstrate that apoA-IV is present in human intestinal epithelial cells and is secreted as a chylomicron and VLDL apoprotein. Within fasting plasma most of the apoA-IV is found free, unassociated

Received for publication 16 July 1979 and in revised form 26 November 1979. with lipoproteins. After lipid ingestion apoA-IV is also found in plasma chylomicrons indicating that some apoA-IV remains associated with chylomicrons in plasma during chylomicron metabolism, although some may be transferred from the chylomicron surface.

\section{INTRODUCTION}

The major apoproteins of plasma lipoproteins have been extensively studied $(1,2)$. In addition to transporting insoluble lipids in body fluids, apoproteins have important roles in lipoprotein metabolism. Apoproteins function as cofactors for enzymes $(3,4)$, bind to cell surface receptors $(5,6)$, regulate intracellular cholesterol content $(5,7)$, and have an important role in the secretion of lipoproteins (8).

Recently the role of the intestine has been explored as a site of synthesis of apoproteins. These studies have revealed that human intestinal epithelium contains apolipoprotein $\mathrm{B}(\mathrm{apoB})^{1}(8,9)$, apoA-I, and apoA-II $(10,11)$ and in vitro isotopic incorporation studies have demonstrated synthesis of these apoproteins (11). Quantitation of apoA-I and apoA-II output in the chylous urine of two subjects with chyluria revealed that the intestine is a major source of these apoproteins for plasma (12).

Several "new" human apoproteins have recently been described. These include apoD (13), apoF (14), and the threonine-poor apoproteins (15). During studies of intestinal lipoproteins performed on two subjects with chyluria resulting from mesenteric lymphatic-urinary fistulae, we noted an apoprotein that was a component of intestinal triglyceride-rich lipoproteins, chylomicrons, and very low density lipoproteins (VLDL) (12). This apoprotein is analogous to a similar rat chylomicron and high density lipoprotein (HDL)

\footnotetext{
${ }^{1}$ Abbreviations used in this paper: $\mathrm{ApoB}$, apolipoprotein $\mathrm{B}$ HDL, high density lipoprotein; LDL, low density lipoproeins; SDS, sodium dodecyl sulfate; VLDL, very low density lipoprotein.
} 
apoprotein, apoA-IV $(16,17)$. Human apoA-IV has been recently described as a component of thoracic duct triglyceride-rich lipoproteins (18) and in plasma chylomicrons from nonfasting hypertriglyceridemic subjects (19). These studies revealed apoA-IV is an immunologically distinct apoprotein with $46,000 \mathrm{~mol}$ wt $(12,18$, 19). We have extended our studies of apoA-IV and demonstrated that human intestinal epithelial cells contain apo-IV and the content increases with lipid absorption consistent with local synthesis. ApoA-IV is transported on chylomicrons and intestinal VLDL and is found in plasma of normal subjects mainly free, unassociated with lipoproteins.

\section{METHODS}

Subjects and collection of samples. The subjects with chyluria have been described previously (12). Urine was collected from one subject at 3-h intervals after the ingestion of $40 \mathrm{~g}$ of corn oil. Sodium azide was added and the samples refrigerated immediately. Aliquots of whole urine were stored at $-80^{\circ} \mathrm{C}$ for apoprotein quantitation. Lipoproteins (chylomicrons, VLDL, and HDL) were isolated from freshly collected samples from both chyluric subjects.

Thoracic duct lymph was collected from a child who had undergone cardiac surgery and had developed a postoperative thoracic duct fistula. The fluid drained continuously from an intrathoracic drainage tube and became milky soon after the ingestion of lipid. Lipoproteins were isolated from the freshly collected fluid.

Blood was collected in the presence of EDTA from five normal male volunteers who had fasted for $18 \mathrm{~h}$ and again $2 \mathrm{~h}$ after the ingestion of one pint of commercially obtained heavy cream. Aliquots of fasting and lipemic plasma were taken for apoprotein, triglyceride, and cholesterol determinations. All human studies were approved by the Human Studies Committee of Presbyterian Hospital, New York. The plasma of four subjects with abetalipoproteinemia, were supplied by Dr. H. J. Kayden (A.M.V. and M.S.) (20), Dr. S. E. Lux (S.S.) (8), and Dr. Claude Roy (N.G.). Plasma from a subject with Tangier disease (10) was supplied by Dr. R. S. Lees.

Isolation and purification of lipoproteins. Lipoproteins were isolated from chylous urine, thoracic duct lymph, and plasma by sequential ultracentrifugation in an L5-65 ultracentrifuge (Beckman Instruments, Inc., Spinco Div., Palo Alto, Calif.). Chylomicrons were isolated in the SW27 rotor by centrifugation at $3 \times 10^{6} \mathrm{~g}$-min and removed by the tube slicer. Other lipoproteins were isolated either in the SW27 or a 40.3 rotor. VLDL were isolated at $10^{8} \mathrm{~g}-\mathrm{min}$, low density lipoprotein (LDL) between the densities $1.006-1.063 \mathrm{~g} / \mathrm{ml}$ and HDL between the densities $1.063-1.21 \mathrm{~g} / \mathrm{ml}(21)$.

Aliquots of unpurified lipoprotein fractions were saved for apoprotein quantification. After initial isolation, chylomicrons and VLDL were purified by passage through $2 \%$ agarose (Biogel A-50M, Bio-Rad Laboratories, Richmond, Calif.) (22). LDL and HDL were purified by recentrifugation at the upper density limit. Lipoproteins were dialyzed against saline to remove the high salt concentration.

Polyacrylamide gel electrophoresis. Purified lipoproteins were delipidated in ethanol ether $(3: 2 \mathrm{vol} / \mathrm{vol})$ at $4^{\circ} \mathrm{C}$ overnight and washed twice with ether. Delipidated apoproteins $(20 \mu \mathrm{g})$ were subjected to sodium dodecyl sulfate (SDS) polyacrylamide gel electrophoresis $(5.6 \%$ gels $)$ in the presence of dithiothreitol as described previously (22). The gels were stained with Coomassie blue. Apoproteins were quantitated densitometrically (22) and were identified by their electrophoretic mobility as compared with purified apoproteins subjected to simultaneous gel electrophoresis. The relative chromogenicity of apoA-I and apoA-IV was determined by subjecting $1-10 \mu \mathrm{g}$ of each apoprotein to SDS gel electrophoresis. The SDS gels were scanned densitometrically and the area under the absorbance tracing quantitated by planimetry. To assess the effect of varying the amount of total protein, 8-30 $\mu \mathrm{g}$ of total chylomicron protein was applied to SDS gels. The percentage distribution of apoproteins remained linear throughout this range of protein.

Isolation of apoA-IV. ApoA-IV was isolated from purified urinary chylomicrons obtained from a chyluric subject. Preparative gel electrophoresis (Polyprep 100, Buchler Instruments, Div. Searle Diagnostics, Inc., Fort Lee, N. J.) was performed using $5.6 \%$ polyacrylamide gel in SDS as described previously (12). Delipidated chylomicron apoproteins were solubilized in $0.2 \mathrm{M}$ Tris buffer, $\mathrm{pH} 7.2$ containing $0.1 \mathrm{M}$ decyl sulphate and $40 \mathrm{mM}$ dithiothreitol before application to the preparative gel. The eluted gel fractions were monitored by analytical SDS gel electrophoresis. The fractions containing pure apoA-IV by SDS gel electrophoresis were extensively dialyzed against $0.05 \mathrm{M} \mathrm{NH}_{4} \mathrm{HCO}_{3}, \mathrm{pH} 8$, and stored at $-20 \mathrm{C}$. Purified apoA-IV did not react with antisera to apoA-I, apoA-II, apoE, or apoB.

Preparation of antisera to apoA-IV. Antisera to apoA-IV were prepared by immunizing rabbits with unfixed and unstained polyacrylamide gel slices of chylomicron apoproteins (23). ApoA-IV was located by its relative mobility as determined on duplicate stained gels scanned densitometrically. Individual gel slices were homogenized, mixed with an equal volume of complete Freund's adjuvant and injected subcutaneously into New Zealand rabbits. The antiserum was characterized by immunodiffusion and immunoelectrophoresis. There was no reaction against purified apoA-I, apoA-II, apoE, or albumin. A single arc of identity was formed against whole serum and purified apoA-IV indicating the antiserum was monospecific.

Immunofluorescence studies on isolated intestinal epithelial cells. Peroral intestinal biopsies were obtained from four normal volunteers aged between 20 and 35 yr. Informed consent was obtained from each subject.

Small-bowel biopsies were performed using a four-port multipurpose Rubin intestinal biopsy tube (Quinton Instruments, Seattle, Wash.). Biopsies were taken from the duodenojejunal junction after an overnight fast. A repeat biopsy was performed $45 \mathrm{~min}$ after $100 \mathrm{ml}$ of heavy cream was instilled into the stomach.

Intestinal epithelial cells were then isolated from the biopsies and indirect immunofluorescence studies performed using antiserum to apoA-IV as previously described $(10,23)$. Nonimmune rabbit serum and antiserum that had been absorbed with purified apoA-IV served as controls. Two observers examined 10 slides from each of the four biopsies. Slides were photographed with Agfachrome 64 film (Eastman Kodak Co., Rochester, N. Y.) with a $\times 40$ oil immersion objective.

Quantitative immunoelectrophoresis of apoA-IV. Quantitation of apoA-IV was performed using the rocket technique of Laurell (24). Electrophoresis was performed using a water cooled electrophoresis apparatus (MRA Corp., Clearwater, Fla.) with a $0.05-\mathrm{M}$ barbital buffer, $\mathrm{pH}$ 8.8. The procedure used was similar to that described previously for apoA-I (10). Antiserum was mixed with $1 \%$ agarose in $0.05 \mathrm{M}$ barbital buffer, pH 8.8 in a final concentration of $5 \%$, and $2 \mu \mathrm{l}$ of sample or standard was applied to each well. In this assay, normal serum gave an identical rocket height if it was: 
(a) diluted with $0.01 \mathrm{M}$ Tris buffer in $8 \mathrm{M}$ urea, $\mathrm{pH} 8$; (b) mixed with an equal volume of 1,1,3,3-tetramethyl-urea and diluted with $0.01 \mathrm{M}$ Tris buffer in $8 \mathrm{M}$ urea, $\mathrm{pH} 8$; or (c) totally delipidated in ethanol ether $(3: 2 \mathrm{vol} / \mathrm{vol})$, washed twice with ether, and then taken up to the desired concentration in $0.01 \mathrm{M}$ Tris buffer in $8 \mathrm{M}$ urea, $\mathrm{pH} 8$. Quantitation of apoA-IV in triglyceride-rich lipoproteins (chylomicrons and intestinal VLDL) required delipidation. When lipemic plasma was assayed, delipidation resulted in $9.6 \%(n=5)$ more apoA-IV being measured, compared with plasma diluted with $0.01 \mathrm{M}$ Tris buffer in $8 \mathrm{M}$ urea. In order to treat all samples identically, total delipidation using ethanol/ether (3:2/vol:vol) was used, the protein precipitate was washed twice with ether, dried under nitrogen, and then taken up in $0.01 \mathrm{M}$ Tris buffer in $8 \mathrm{M}$ urea. Serum (usually $25 \mu \mathrm{l}$ ) was delipidated then the protein precipitate taken up in $200 \mu \mathrm{l}$ of Tris urea buffer for a final dilution of $1: 8$. By this method larger volumes $(200 \mu \mathrm{l})$ of lipoprotein fractions were delipidated and the protein precipitate taken up in smaller volumes $(50 \mu \mathrm{l})$ of buffer allowing detection of small amount of apoA-IV in lipoprotein fractions. Purified apoA-IV in $0.01 \mathrm{M}$ Tris buffer in $8 \mathrm{M}$ urea was used as a standard.

Column chromatography. Column chromatography of whole plasma in $10 \%$ agarose (Biogel A 50M, 100-200 mesh) was performed with two $120 \times 1.0-\mathrm{cm}$ columns run in series. The columns were eluted using $\mathrm{NaCl}$-EDTA buffer at $4^{\circ} \mathrm{C}$ at a flow rate of $2.5 \mathrm{ml} / \mathrm{h}$. Sample volumes of $0.5 \mathrm{ml}$ were applied to the column. The column was calibrated with plasma LDL and HDL. ApoA-I and apoB were detected in column fractions by Ouchterlony immunodiffusion. Fractions were pooled, dialyzed against water, and lyophilized. The lyophilized fractions were delipidated in chloroform:methanol and washed twice in ether. The delipidated fractions were dissolved in small volumes of Tris/urea buffer and subjected to rocket immunoelectrophoresis in order to quantitate apoA-IV. The chloroform:methanol lipid extract was subject to thin-layer chromatography to detect the presence of lipid $(25,26)$.

Triglyceride and cholesterol were determined by the autoanalyzer technique (27) and proteins by the method of Lowry et al. (28).

\section{RESULTS}

SDS gel electrophoresis. Chylomicrons, VLDL, and HDL were isolated from thoracic duct lymph and chylous urine and purified as described in Methods. The SDS gels of the thoracic duct lipoproteins are shown in Fig. 1. The lipoproteins from chylous urine had a similar gel pattern (Fig. 4) (12). The distribution of apoproteins was assessed by planimetry of SDS gels and is shown in Table I. The values represent the percentage distribution of area under the absorbance tracing. Studies performed to assess the relative chromagenicity of apoA-I and apoA-IV revealed that the area under the absorbance tracing for each protein was linear between 1 and $10 \mu \mathrm{g}$ of protein. The relative chromagenicity of apoA-I and apoA-IV did not differ by $>10 \%$ over this range of protein. Chylomicrons and VLDL from both sources contained similar apoproteins including apoB, apoA-IV, apoE, apoA-I, apoC, and apoA-II. The $\mathrm{C}$ proteins and apoA-II migrate together in this reduced gel system, however, apoA-II was detected immunologically in chylomicrons and VLDL

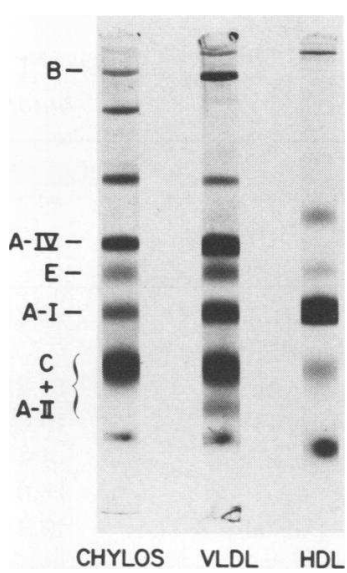

FIGURE 1 SDS gels of thoracic duct lymph lipoproteins. Chylomicrons, VLDL, and HDL were isolated from fatty lymph and purified as in Methods. Apoprotein bands are labeled. The ink marker indicating the electrophoresis front is seen at the bottom of the gel.

from both sources. ApoA-IV comprised 10-13\% of chylomicron protein and a greater percentage $(24 \%$ and $30 \%$ ) of urinary VLDL and thoracic duct VLDL, respectively. The chylomicrons and VLDL were generally free of albumin by Ouchterlony analysis using antisera to human albumin. Unidentified protein bands of higher molecular weight were also present. A major band of $R_{\mathrm{f}}=0.35(\sim 100,000 \mathrm{~mol} \mathrm{wt})$ was noted in chylomicrons and VLDL from thoracic duct lymph. This protein was noted previously in chylomicrons from chylous urine (12) and has not been further identified. The gels of HDL from both sources contained no detectable apoA-IV.

Immunofluorescence. Intestinal biopsies were obtained from four volunteers after an overnight fast and again $45 \mathrm{~min}$ after cream was instilled into the stomach. Four biopsies were taken on each occasion. Intestinal epithelial cells were isolated from each biopsy. Cells prepared from fasting biopsies demonstrated limited immunofluorescence consistent with the presence of only small amounts of intracellular apoA-IV (Fig. 2, left). $45 \mathrm{~min}$ after the ingestion of cream, apoA-IV fluorescence in the apical portion of cells markedly increased, suggesting an increase in apoA-IV content during fat absorption (Fig. 2, right). All fluorescence was blocked by prior absorption of the antiserum with purified apoA-IV. Identical results were obtained in each of four normal subjects.

Electroimmunoassay of apoA-IV. To quantitate apoA-IV an electroimmunoassay was developed. All samples were totally delipidated for this assay. Dilutions of normal sera gave a linear response when run in the assay. The apoA-IV standard was linear between $0.75 \mathrm{mg} / \mathrm{dl}$ and $8 \mathrm{mg} / \mathrm{dl}(r=0.9992)$. The lowest point of linearity corresponded to $15 \mathrm{ng}$ of standard 
TABLE I

Apopxetein Content of Chylomicrons and Intestinal VLDL from

Chylous Urine, Thoracic Duct Lymph, and Plasma

\begin{tabular}{|c|c|c|c|c|c|}
\hline & \multicolumn{2}{|c|}{ Chylous urine* } & \multicolumn{2}{|c|}{ Thoracic duct lymph } & \multirow{2}{*}{$\begin{array}{c}\text { Plasma } \\
\begin{array}{c}\text { Chylos } \\
n=5\end{array}\end{array}$} \\
\hline & $\begin{array}{c}\text { Chylos } \\
n=7\end{array}$ & $\begin{array}{l}\text { VLDL } \\
n=5\end{array}$ & $\begin{array}{l}\text { Chylos } \\
n=3\end{array}$ & $\begin{array}{l}\text { VLDL } \\
n=1\end{array}$ & \\
\hline ApoB & $3.4 \pm 0.7$ & $10.9 \pm 1.9$ & $9.8 \pm 0.2$ & 9.7 & $17.5 \pm 3.3$ \\
\hline $\begin{array}{l}\text { Unidentified } \\
\text { protein }\end{array}$ & $13.8 \pm 3.0$ & $8.3 \pm 0.8$ & $10.0 \pm 0.8$ & - & $7.0 \pm 2.3$ \\
\hline ApoA-IV & $10.0 \pm 3.3$ & $24.6 \pm 2.8$ & $13.0 \pm 0.5$ & 30.0 & $4.9 \pm 2.1$ \\
\hline ApoE & $4.4 \pm 0.3$ & $6.0 \pm 0.3$ & $7.3 \pm 0.1$ & 6.0 & $17.4 \pm 1.4$ \\
\hline ApoA-I & $15.0 \pm 1.8$ & $16.5 \pm 2.0$ & $10.4 \pm 0.2$ & 15.5 & $1.9 \pm 0.9$ \\
\hline ApoC and A-II & $49.3 \pm 11.3$ & $30.3 \pm 3.5$ & $47.1 \pm 2.2$ & 35.1 & $48.5 \pm 4.2$ \\
\hline
\end{tabular}

Chylomicrons and VLDL were isolated by ultracentrifugation and purified by agarose column chromatography. The lipoproteins were delipidated and subjected to polyacrylamide gel electrophoresis. Apoproteins were quantitated by planimetry of densitometric gel scans. The values represent the percentage distribution of area under the absorbance tracing.

Values are mean $\pm S E M$ of the percentage area under each apoprotein peak.

* Chylomicrons and VLDL were isolated from the urine of two subjects with chyluria.

f Obtained from thoracic duct fistula draining through the pleural cavity.

applied to the well. Normal serum apoA-IV was $15.7 \pm 0.9 \mathrm{mg} / \mathrm{dl}( \pm \mathrm{SEM}), n=14$. Intra- and inter-assay coefficients of variations were 4 and $8.2 \%$, respectively. The four subjects with abetalipoproteinemia had reduced levels of apoA-IV: A.M.V., $9.6 \mathrm{mg} / \mathrm{dl}$; M.S., 7.6 $\mathrm{mg} / \mathrm{dl}$; S.S., $1.2 \mathrm{mg} / \mathrm{dl}$; and N.G., $8.3 \mathrm{mg} / \mathrm{dl}$.
Quantification of apoA-IV output in chylous urine after lipid ingestion. One subject with chyluria was given $40 \mathrm{~g}$ of corn oil orally. Urine was collected at 3-h intervals and triglyceride and apoA-IV were measured (Fig. 3). Urinary triglyceride rose rapidly. Over $15 \mathrm{~h} 11.5 \mathrm{~g}$ of triglyceride was excreted representing
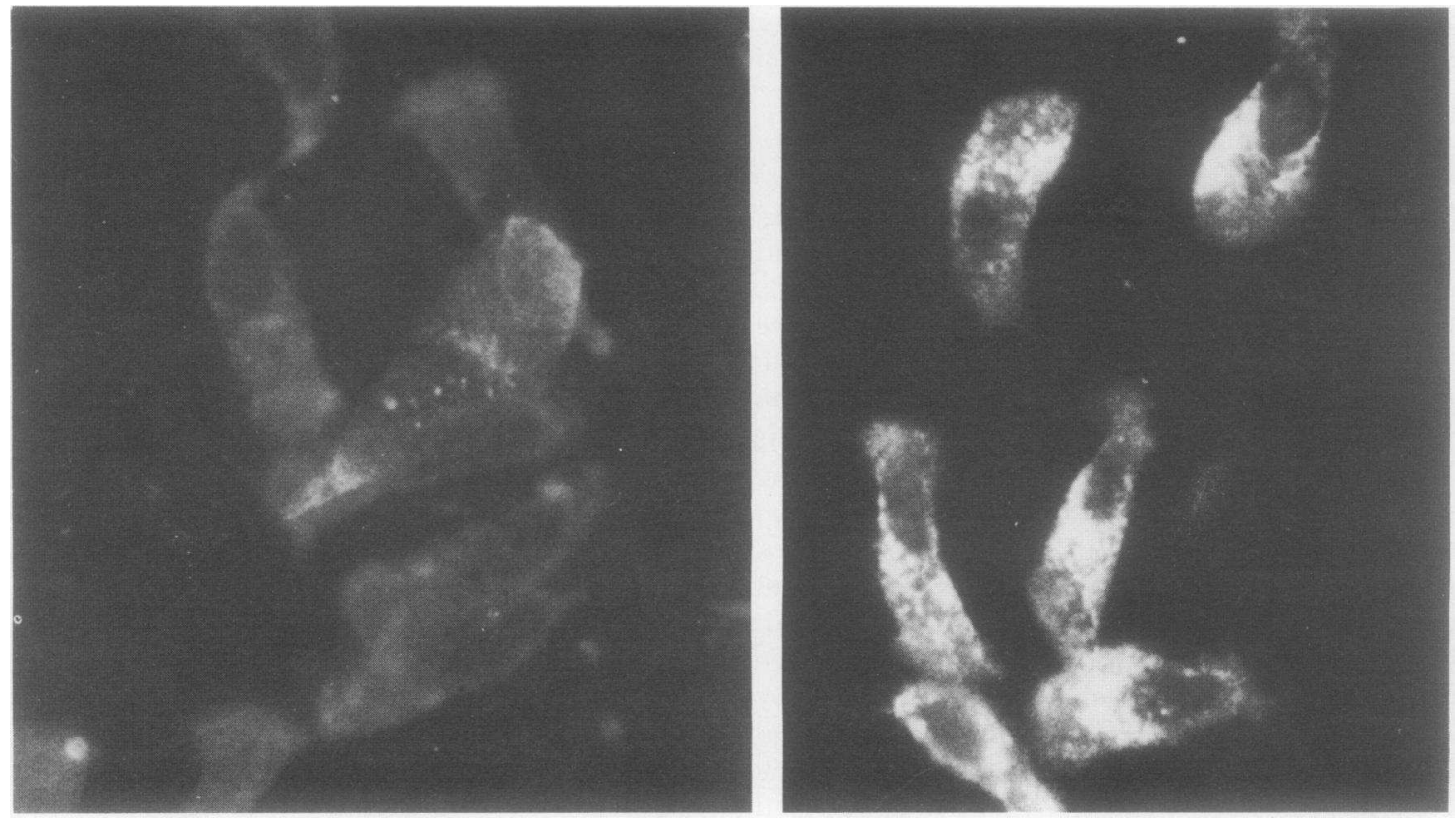

FIGURE 2 Immunofluorescence for apoA-IV. Epithelial cells were isolated from intestinal biopsies taken while fasting (left) and during lipid absorption (fat-fed, right). Indirect immunofluorescence for apoA-IV was carried out (Methods). 


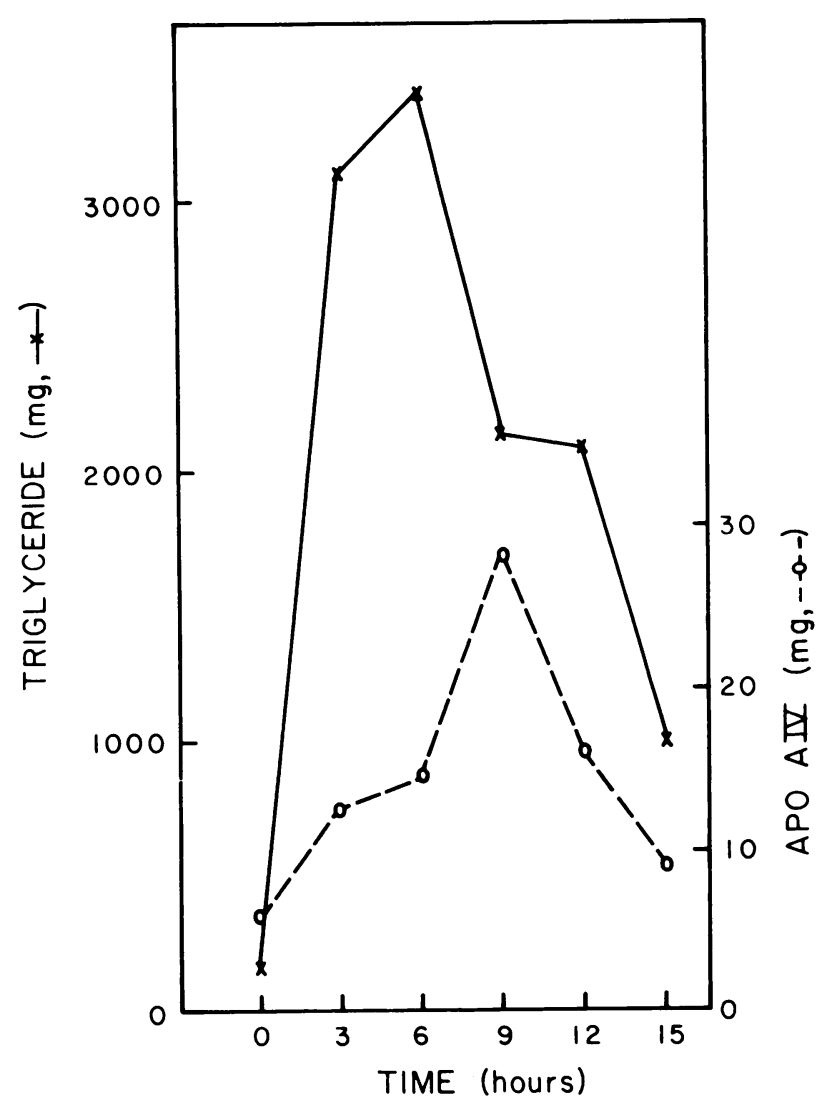

FIGURE 3 Output of triglyceride and apoA-IV in chylous urine. The chyluric subject ingested $40 \mathrm{~g}$ corn oil, urine was collected at 3-h intervals. Triglyceride and apoA-IV were quantified as in Methods. The zero time value is for the 3-h period before fat administration.

$28 \%$ of the ingested lipid. This percent of lipid secreted is considered to represent the percent of mesenteric lymph shunted into urine (29). Output of apoA-IV in urine increased along with triglyceride, consistent with apoA-IV secretion by the intestine and transport in mesenteric lymph during chylomicron and VLDL formation. The peak output of apoA-IV and triglyceride were dysynchronous. The reason for this is not clear. Over $15 \mathrm{~h}, 81 \mathrm{mg}$ of A-IV was excreted in the urine.

Aliquots of whole urine were subjected to sequential ultracentrifugation to remove chylomicrons and VLDL. Quantification of apoA-IV was performed on the unwashed chylomicron and VLDL fraction as well as the $d>1.006-\mathrm{g} / \mathrm{ml}$ fraction (Table III). $56.3 \%$ was present in the triglyceride-rich, $d<1.006-\mathrm{g} / \mathrm{ml}$ fraction, whereas $43.2 \%$ was present in the $d>1.006-\mathrm{g} / \mathrm{ml}$ fraction.

Effect of alimentary lipemia on plasma apoA-IV concentration. To determine whether the increased content of apoA-IV in intestinal epithelial cells and the increased amount of apoA-IV transported in mesenteric lymph could contribute to plasma levels, apoA-IV was measured in plasma during alimentary lipemia. Blood was taken from five normal volunteers in the fasting state and after ingestion of lipid. As shown in Table II the ingestion of cream by five normal volunteers resulted in a rise in plasma triglyceride and significant rise of $16 \%$ in plasma apoA-IV levels. ApoA-IV rose from $16.1 \pm 0.7 \mathrm{mg} / \mathrm{dl}$ to $18.5 \pm 0.7 \mathrm{mg} / \mathrm{dl}(n=5, P<0.01)$.

Distribution of apoA-IV in plasma. Lipoproteins were isolated from fasting and lipemic plasma from each of the normal volunteers. Before purification aliquots of the lipoprotein fractions were taken for quantitative electroimmunoassay for apoA-IV. Lipoproteins were then purified (see Methods), delipidated, and subject to SDS gel electrophoresis. The SDS gels of lipoproteins from the lipemic plasma of one volunteer are shown in Fig. 4 and compared to urinary chylomicrons. ApoA-IV was detected in the purified plasma chylomicrons (Svedberg units of flotation $\left[\mathrm{S}_{\mathrm{f}}\right]$ $>400$ ) from four of the five subjects. The apoprotein content (as assessed by densitometric scans of SDS gels) is shown in Table I. ApoA-IV represented $4.9 \%$ of purified chylomicron protein. No apoA-IV was detected in other plasma lipoproteins from fasting or lipemic plasma by this technique.

The distribution of apoA-IV in unwashed lipoproteins from fasting and lipemic plasma was assessed by electroimmunoassay (Table III). Unwashed lipoproteins were used because repetitive ultracentrifugation results in loss of apoproteins (17) and we have noted that centrifugation of chylous urine chylomicrons for up to $72 \mathrm{~h}$ results in almost complete loss of apoA-IV (unpublished results). In contrast to chylous urine in which $56 \%$ of apoA-IV is associated with triglyceriderich lipoproteins, only $1 \%$ of fasting plasma apoA-IV was present in the $d<1.006$ fraction. In lipemic plasma the $d<1.006$ fraction contained $10 \%$ of the plasma apoA-IV. This was mainly accounted for by apoA-IV in the chylomicron fraction. The majority of apoA-IV was present in the lipoprotein-free fraction of plasma $(d>1.21 \mathrm{~g} / \mathrm{ml})$. HDL contained $<1.0 \%$ of the

TABLE II

Human Plasma ApoA-IV Alimentary Lipemia

\begin{tabular}{lcc}
\hline & Pre & Post \\
\hline & \multicolumn{2}{c}{$m g / d l^{*}$} \\
Triglyceride & $77 \pm 10$ & $214 \pm 46 \ddagger$ \\
Cholesterol & $185 \pm 12$ & $185 \pm 14$ \\
ApoA-IV & $16.1 \pm 0.7$ & $18.5 \pm 0.7 \ddagger$
\end{tabular}

Blood was drawn from five fasting normal volunteers and again after the ingestion of one pint of cream. Triglyceride, cholesterol, and apoA-IV were quantified as in Methods.

* Mean $\pm \operatorname{SEM}(n=5)$.

$\$$ By paired $t$ test $P<0.01$. 


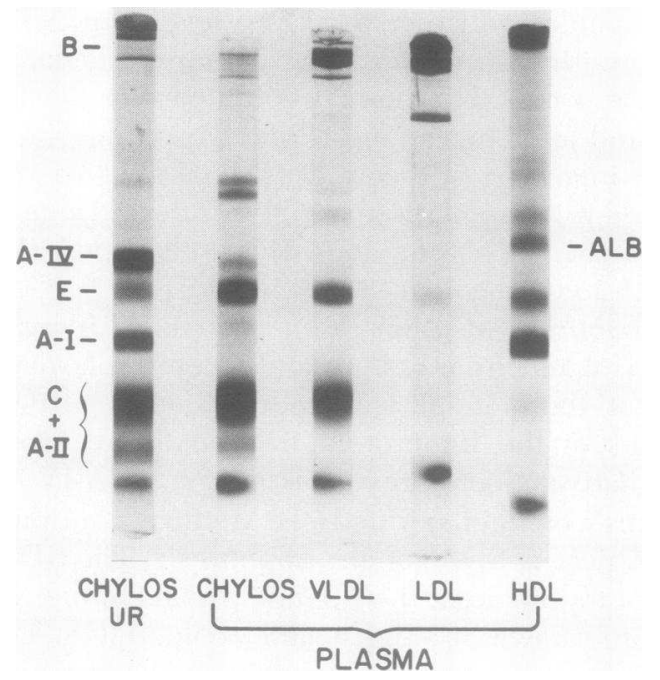

FIGURE 4 SDS gels of chylomicrons from chylous urine and lipoproteins from lipemic plasma obtained from one subject after the ingestion of one pint of cream. Lipoproteins were purified as in Methods.

total plasma apoA-IV. This small amount appears to be associated with the lipoprotein and not present as a result of contamination with small amounts of the $d>1.21 \mathrm{~g} / \mathrm{ml}$ fraction as small amounts of apoA-IV were detected immunologically in purified (recentrifuged) HDL. ApoA-IV only accounted for $0.05 \%$ $(0.05 \pm 0.01 \%, n=4)$ of purified HDL protein.

The occurrence of apoA-IV in the $d>1.21$ fraction of serum might be the result of ultracentrifugation or due to the apoprotein existing free in plasma. To further explore this possibility, immunoelectrophore-

TABLE III

Percentage Distribution of APO A-IV in Chylous Urine and Plasma Lipoproteins

\begin{tabular}{lccc}
\hline & $\begin{array}{c}\text { Chylous } \\
\text { urine } \\
n=5\end{array}$ & $\begin{array}{c}\text { Fasting } \\
\text { plasma } \\
n=5\end{array}$ & $\begin{array}{c}\text { Lipemic } \\
\text { plasma } \\
n=5\end{array}$ \\
\hline Chylomicrons & $41.7 \pm 6.2$ & - & $8.8 \pm 1.4$ \\
VLDL & $14.6 \pm 1.7$ & $1.0 \pm 0.6$ & $1.2 \pm 0.5$ \\
LDL & & $0.3 \pm 0.2$ & ND* \\
HDL & & $0.7 \pm 0.1$ & $0.5 \pm 0.2$ \\
$\begin{array}{l}\text { H }>1.21 \\
\text { T }\end{array}$ & $43.2 \pm 5.9$ & $98.1 \pm 0.1$ & $89.8 \pm 1.3$ \\
\hline
\end{tabular}

Chylous urine was collected from two subjects after oral administration of corn oil. Plasma was collected from five volunteers before and after the ingestion of one pint of cream and subject to sequential ultracentrifugation. ApoA-IV was measured in unpurified fractions. Values are the percentage distribution of apoA-IV in lipoprotein fractions. Mean recovery of apoA-IV in the combined lipoprotein fractions was 90,89 , and $88 \%$ for chylous urine, fasting plasma, and lipemic plasma, respectively.

* ND, not detected. sis of whole plasma and the $d>1.21$ fraction was performed. The immunoprecipitate arc of apoA-IV from fasting whole serum and the lipoprotein-free fraction $(d>1.21 \mathrm{~g} / \mathrm{ml})$ were in identical positions which was between the pre- $\beta$ and $\alpha$ positions and did not correspond to the mobility of plasma lipoproteins. The results suggest that the bulk of apoA-IV detectable by this method in plasma is free. Similar findings were reported by Beisiegel and Utermann (19).

Whole fasting plasma from one subject was fractionated by agarose column chromatography using $10 \%$ agarose. The elution profile is shown in Fig. 5. ApoB and apoA-I were detected in the column fractions by Ouchterlony immunodiffusion. The elution volumes of LDL and HDL are shown. These elution volumes corresponded with the localization of apoB and apoA-I. Pooled fractions were dialyzed, lyophilized, delipidated, and solubilized in Tris-urea buffer for immunoassay for apoA-IV. The majority (77\%) of apoA-IV was present in the fractions that eluted after HDL. This fraction contained only small amounts of phospholipid. Total recovery of apoA-IV from the column was $85 \%$ of that applied.

\section{DISCUSSION}

ApoA-IV is an immunologically distinct human apoprotein analogous to a similar rat apoprotein. Studies in the rat have demonstrated synthesis of apoA-IV by the intestine $(30,31)$ and to a lesser extent by the liver (32). In rat mesenteric lymph apoA-IV is found as a chylomicron, intestinal VLDL, and HDL apoprotein $(31,33)$, as well as free in the $d>1.21-\mathrm{g} / \mathrm{ml}$ fraction (31). In rat plasma, apoA-IV is found in both the HDL and $d>1.21$ fraction $(16,17,34)$ and is possibly transferred from the chylomicron surface, together with phospholipid and apoA-I into HDL during chylomicron metabolism (35).

ApoA-IV has been isolated from intestinal triglyceride-rich lipoproteins obtained from chylous urine (12) and from human thoracic duct lymph (18). Characterization has demonstrated that it is an immunologically distinct human apoprotein, with a molecular weight of 46,000. Beisiegel and Utermann (19) isolated apoA-IV from the nonfasting plasma of several hypertriglyceridemic subjects. They demonstrated that apoA-IV was present on plasma chylomicrons, however it was easily lost from the chylomicron surface during ultracentrifugation. It was also found by Ouchterlony immunodiffusion in the $d>1.21 \mathrm{~g} / \mathrm{ml}$ fraction of plasma.

To explore the role of the human intestine in apoA-IV metabolism we studied lipoproteins from chylous urine, human thoracic duct lymph, and the plasma of normal volunteers during alimentary lipemia. We also performed immunofluorescence studies 


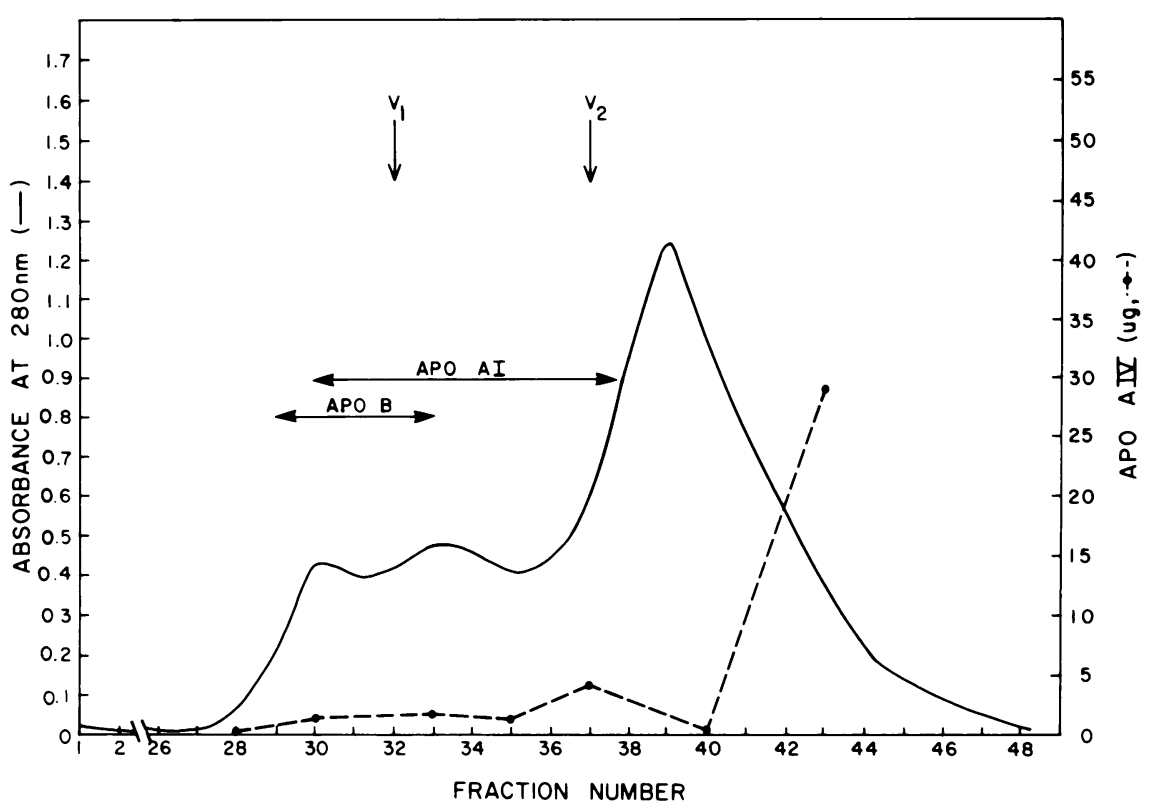

FIGURE 5 Agarose column chromatography of fasting plasma. $V_{1}$ and $V_{2}$ represent the elution volumes of LDL and HDL. ApoA-I and apoB were detected in fractions by Ouchterlony immunodiffusion. ApoA-IV was measured in pooled fractions by rocket immunoelectrophoresis.

on isolated intestinal epithelial cells from normal volunteers.

The findings of limited intracellular fluorescence for apoA-IV in intestinal epithelial cells from fasting subjects and a marked increase in fluorescence during lipid absorption is consistent with synthesis of apoA-IV by the intestine during lipid absorption. This is supported by the marked increase in apoA-IV excreted in the urine of one chyluric subject after lipid ingestion, and the small but statistically significant rise in plasma apoA-IV levels during alimentary lipemia in normal volunteers.

In chylous urine $56 \%$ of apoA-IV was associated with triglyceride-rich lipoproteins presumably secreted by the intestine. We cannot be sure of the origin of that present in the $d>1.006$ fraction. Some apoA-IV may be present in this fraction as a result of displacement from less dense lipoproteins due to ultracentrifugation. The contribution of free apoA-IV filtered from plasma during lipid absorption is also not known.

To estimate the amount of apoA-IV secreted by the intestine during lipid absorption, calculations based on the extent of the mesenteric lymphatic shunt were performed. In addition it is assumed that only the apoA-IV associated with triglyceride-rich lipoproteins (chylomicrons and intestinal VLDL) was derived from the intestine. These calculations reveal that for $40 \mathrm{~g}$ of ingested lipid, $160 \mathrm{mg}$ of apoA-IV was secreted on chylomicrons and intestinal VLDL. We cannot, however, estimate the intestinal contribution of apoA-IV to plasma levels as we do not know the plasma resi- dence time. It is also not known whether the human liver secretes apoA-IV.

ApoA-IV was found in normal plasma in a concentration of $15.7 \mathrm{mg} / \mathrm{dl}$. Using three different techniques the bulk of fasting plasma apoA-IV was found free, unassociated with lipoproteins. Ultracentrifugally, $98 \%$ of apoA-IV was found in the $d>1.21-\mathrm{g} / \mathrm{ml}$ fraction whereas $77 \%$ was found in this fraction using column chromatography. The remainder $(27 \%)$ eluted with lipoproteins suggesting that small amounts of apoA-IV do exist associated wth lipoproteins. This was confirmed by the findings of very small amounts of apoA-IV in purified plasma HDL $(<0.1 \%$ of HDL protein) using immunological techniques, despite the absence of apoA-IV on SDS gels. Although the majority of apoA-IV was found in the lipoprotein-free fraction of plasma, the present studies have not determined whether this apoprotein is associated with small amounts of lipid (i.e., phospholipid) or indeed exists in a lipid-free form in plasma. Studies are in progress to more fully characterize the apoA-IV in this fraction.

After the ingestion of lipid, plasma apoA-IV levels rose by $16 \%$. The rise was seen in each subject studied, indicating that intestinal apoA-IV contributes to plasma levels. Chylomicrons were isolated from plasma by a single ultracentrifugation and purified by agarose column chromatography. SDS gels of chylomicrons from four of the five subjects studied revealed the presence of apoA-IV. The unpurified $d<1.006$ fraction (Table III) contained $10 \%$ of plasma apoA-IV indicating that in lipemic plasma a significant amount 
of apoA-IV is still associated with chylomicrons. The bulk $(90 \%)$ however, was recovered in the $d>1.21$ fraction.

These studies are consistent with apoA-IV synthesis in the human intestine and secretion as a chylomicron and intestinal VLDL component. The findings of reduced plasma levels of apoA-IV in four subjects with abetalipoproteinemia who cannot form chylomicrons or VLDL $(36,37)$ indicate that apoA-IV may be secreted independently of this mechanism. Within plasma, apoA-IV exists associated with plasma chylomicrons, small amounts in other lipoproteins, and the majority free, unassociated with lipoproteins. During in vitro incubation studies using human chylomicrons (12) apoA-IV and apoA-I were transferred from the chylomicron surface when chylomicrons were incubated with plasma or HDL. ApoA-IV was not however, recovered in HDL reisolated from the incubation mixture by ultracentrifugation. These studies suggest that HDL may be involved in the subsequent metabolism of human apoA-IV even though it is not a major HDL apoprotein in normal plasma. It is of interest that apoA-IV levels determined in one patient with Tangier disease $(10,38)$ were normal $(13.6 \mathrm{mg} / \mathrm{dl})$ despite severely reduced HDL and apoA-I levels (10) indicating that apoA-IV does not participate in the abnormal catabolism of chylomicron-HDL apoproteins characteristic of Tangier disease $(39,40)$. In addition, we have observed that some subjects with acquired lecithin cholesterol acyltransferase deficiency resulting from alcoholic hepatitis have apoA-IV as an HDL apoprotein (unpublished results) again suggesting that HDL may be involved in apoA-IV metabolism. The present studies have not delineated the mechanisms whereby apoA-IV originating in the intestine is metabolized in the systemic circulation. It is probable that some apoA-IV may transfer from the surface of intestinal lipoproteins to other plasma lipoproteins (e.g., HDL) during lipolysis, along with other chylomicron surface components $(35,41)$. Further studies are necessary to determine the role of plasma lipoproteins in apoA-IV metabolism and the factors that determine its ultimate distribution in plasma.

\section{ACKNOWLEDGMENTS}

This work was supported by National Institutes of Health grants A.M 21367-02 and HL 21006-03 Scope F.

\section{REFERENCES}

1. Eisenberg, S., and R. I. Levy. 1975. Lipoprotein metabolism. Adv. Lipid Res. 13: 1-89.

2. Schaefer, E. J., S. Eisenberg, and R. I. Levy. 1978. Lipoprotein apoprotein metabolism. J. Lipid Res. 19: 667-687.

3. Fielding, C. J., V. G. Shane, and P. E. Fielding. 1972.
A protein cofactor of lecithin: cholesterol transference. Biochem. Biophys. Res. Commun. 46: 1493-1498.

4. LaRosa, J. C., R. I. Levy, P. N. Herbert, S. E. Lux, and D. S. Fredrickson. 1970. A specific apoprotein activator for lipoprotein lipase. Biochem. Biophys. Res. Commun. 41: 57-62.

5. Goldstein, J. L., and M. S. Brown. 1977. The low density lipoprotein pathway and its relation to arteriosclerosis. Annu. Rev. Biochem. 46: 897-930.

6. Innerarity, T., and R. W. Mahley. 1978. Enhanced binding by cultured human fibroblasts of apo-E containing lipoproteins as compared with low density lipoproteins. Biochemistry. 17: 1440-1447.

7. Stein, Y., M. C. Glangleaud, M. Fainaru, and O. Stein. 1975. The removal of cholesterol from aortic smooth muscle cells in culture and landschutz ascites cells by fractions of human high density apolipoprotein. Biochim. Biophys. Acta. 380: 106-118.

8. Glickman, R. M., P. H. R. Green, R. S. Lees, S. E. Lux, and A. Kilgore. 1979. Immunofluorescence studies of apolipoprotein B in intestinal mucosa: absence in abetalipoproteinemia. Gastroenterology. 76: 288-292.

9. Rachmilewitz, D., J. J. Albers, and D. R. Saunders. 1976. Apoprotein B in fasting and postprandial human jejunal mucosa. J. Clin. Invest. 57: 530-533.

10. Glickman, R. M., P. H. R. Green, R. S. Lees, and A. R. Tall. 1978. Apoprotein A-I synthesis in normal intestinal mucosa and in Tangier disease. N. Engl. J. Med. 299: 1424-1427.

11. Rachmilewitz, D., J. J. Albers, and D. R. Saunders. 1978. Apoprotein synthesis by human duodenojejunal mucosa. Gastroenterology. 75: 667-682.

12. Green, P. H. R., R. Glickman, C. D. Saudek, C. B. Blum, and A. R. Tall. 1979. Human intestinal lipoproteins. Studies in chyluric subjects. J. Clin. Invest. 64: 233-242.

13. Curry, M. D., W. J. McConathy, and P. Alaupovic. 1977. Quantitative determination of human apolipoprotein D by electroimmunoassay and radial immunodiffusion. Biochim. Biophys. Acta. 491: 232-241.

14. Olofsson, S. O., W. J. McConathy, and P. Alaupovic. 1978. Isolation and partial characterization of a new acidic apolipoprotein (apolipoprotein F) from high density lipoproteins of human plasma. Biochemistry. 17: $1032-1036$.

15. Shore, V. G., B. Shore, and S. B. Lewis. 1978. Isolation and characterization of two threonine-poor apolipoproteins of human plasma high density lipoproteins. Biochemistry. 17: 2174-2179.

16. Swaney, J. B., F. Braithwaite, and H. A. Eder. 1977. Characterization of the apolipoproteins of rat plasma lipoproteins. Biochemistry. 16: 271-278.

17. Imaizumi, K., M. Fainaru, and R. Havel. 1978. Composition of proteins of mesenteric chylomicrons in the rat and alterations on exposure of chylomicrons to blood serum and serum proteins. J. Lipid Res. 19: 712-722.

18. Weisgraber, K. H., T. P. Bersot, and R. W. Mahley. 1978. Isolation and characterization of an apoprotein from the $d>1.006$ lipoproteins of human and canine lymph homologous with the rat A-IV apoprotein. Biochem. Biophys. Res. Commun. 85: 287-292.

19. Beisiegel, U., and G. Utermann. 1979. An apolipoprotein homolog of rat apolipoprotein A-IV in human plasma: isolation and partial characterization. Eur. J. Biochem. 93: $601-608$.

20. Scanu, A. M., L. P. Aggerbeck, A. W. Kruski, C. T. Lim, and H. J. Kayden. 1974. A study of the abnormal lipoproteins in abetalipoproteinemia. J. Clin. Invest. 53: 440-453. 
21. Havel, R. J., H. A. Eder, and J. H. Bragdon. 1955. The distribution and chemical composition of ultracentrifugally separated lipoproteins in human serum. J. Clin. Invest. 34: 1345-1353.

22. Glickman, R. M., and K. Kirsch. 1973. Lymph chylomicron formation during the inhibition of protein synthesis: Studies of chylomicron apoproteins. J. Clin. Invest. 52: 2910-2920.

23. Glickman, R. M., J. Khorana, and A. Kilgore. 1976. Localization of apolipoprotein B in intestinal epithelial cells. Science (Wash. D. C.). 193: 1254-1255.

24. Laurell, C. B. 1966. Quantitative estimation of proteins by electrophoresis in agarose gel containing antibodies. Anal. Biochem. 15: 45-52.

25. Downing, D. T. 1968. Photodensitometry in the thin-layer chromatographic analysis of neutral lipids.J. Chromatogr. 38: $91-99$.

26. Katz, S. S., G. G. Shipley, and D. M. Small. 1976. Physical chemistry of the lipids of human atherosclerotic lesions. J. Clin. Invest. 58: 200-211.

27. Kessler, G., and M. Lederer. 1966. Fluorometric measurement of triglyceride. Automation in Analytical Chemistry: Technicon Symposium. Technicon Laboratories, Tarrytown, N. Y. 341-344.

28. Lowry, O. M., N. J. Rosebrough, A. L. Farr, and R. J. Randall. 1951. Protein measurement with the Folin phenol reagent. J. Biol. Chem. 193: 265-275.

29. Blomstrand, R., N. A. Thorn, and E. H. Ahrens, Jr. 1958. The absorption of fats, studied in a patient with chyluria. Am. J. Med. 24: 958-966.

30. Imaizumi, K., R. J. Havel, M. Fainaru, and J. L. Vigne. 1978. Origin and transport of the A-I and arginine-rich apolipoproteins in mesenteric lymph of rats. J. Lipid Res. 19: $1038-1046$

31. Wu, A. L., and H. G. Windmueller. 1978. Identification of circulating apolipoproteins synthesized by rat small intestine in vivo. J. Biol. Chem. 253: 2525-2528.

32. Wu, A. L. and H. G. Windmueller. 1978. Relative contribution by liver and intestine to individual plasma apoproteins in the rat. Circulation. 58: 11-15

33. Green, P. H. R., A. R. Tall, and R. M. Glickman. 1978. Rat intestine secretes discoid high density lipoprotein. J. Clin. Invest. 61: 528-534.

34. Roheim, P. S., D. Edelstein, and G. G. Pinter. 1976. Apolipoproteins in rat serum and renal lymph. Proc. Natl. Acad. Sci. U. S. A. 73: 1757-1760.

35. Tall, A. R., P. H. R. Green, R. M. Glickman, and J. W. Riley. 1979. Metabolic fate of chylomicron phospholipids and apoproteins in the rat. J. Clin. Invest. 64: 977-989.

36. Gotto, A. M., R. I. Levy, K. John, and D. S. Frederickson. 1971. On the protein defect in abetalipoproteinemia. N. Engl. J. Med. 284: 813-818.

37. Kayden, H. J. 1972. Abetalipoproteinemia. Annu. Rev. Méd. 23: 285-296.

38. Brook, J. G., R. S. Lees, J. H. Yules, and B. Cusack. 1977. Tangier disease ( $\alpha$-lipoprotein deficiency). JAMA (J.Am. Med. Assoc.). 238: 332-334.

39. Schaefer, E. J., C. B. Blum, R. I. Levy, H. B. Brewer, R. Goebel, and M. Berman. 1978. High density lipoprotein metabolism in Tangier disease. N. Engl. J. Med. 299: 905-910.

40. Schaefer, E. J., and H. B. Brewer, Jr. 1978. Tangier disease: a defect in the conversion of chylomicrons to high density lipoproteins. Clin. Res. 26: 532A. (Abstr.)

41. Redgrave, T. G., and D. M. Small. 1979. Quantitation of the transfer of surface phospholipid of chylomicrons to the high density lipoprotein fraction during the catabolism of the chylomicrons. J. Clin. Invest. 64: $162-171$. 